\title{
THE EFFICIENCY OF STANDARD WREATH PRODUCT
}

\author{
A. SINAN CEVIK \\ Balikesir Universitesi, Fen-Edebiyat Fakultesi, \\ Matematik Bolumu, 10100 Balikesir, Turkey
}

(Received 5 September 1998)

\begin{abstract}
Let $\xi$ be the set of all finite groups that have efficient presentations. In this paper we give sufficient conditions for the standard wreath product of two $\xi$-groups to be a $\xi$-group.
\end{abstract}

Keywords: standard wreath product; efficiency; presentations

AMS 1991 Mathematics subject classification: Primary 20E22; 20F05

\section{Introduction}

\section{(a) Efficiency}

Let $G$ be a finitely presented group, and let $\mathcal{P}=\langle\boldsymbol{x} ; \boldsymbol{r}\rangle$ be a finite presentation for $G$. The deficiency of $\mathcal{P}$ is defined by $\operatorname{def}(\mathcal{P})=-|x|+|r|$. Let

$$
\delta(G)=-r k_{\mathbb{Z}}\left(H_{1}(G)\right)+d\left(H_{2}(G)\right),
$$

where $r k_{\mathbb{Z}}(\cdot)$ denotes the $\mathbb{Z}$-rank of the torsion-free part and $d(\cdot)$ means the minimal number of generators. Then it is known (see $[\mathbf{5}, \mathbf{8}, \mathbf{1 2}]$ ) that for the presentation $\mathcal{P}$, it is always true that $\operatorname{def}(\mathcal{P}) \geqslant \delta(G)$. We define

$$
\operatorname{def}(G)=\min \{\operatorname{def}(\mathcal{P}): \mathcal{P} \text { a finite presentation for } G\}
$$

We say $G$ is efficient if $\operatorname{def}(G)=\delta(G)$, and a presentation $\mathcal{P}$ such that $\operatorname{def}(\mathcal{P})=\delta(G)$ is then called an efficient presentation.

\section{(b) Known results}

Examples of efficient groups are finitely generated abelian groups, fundamental groups of closed 3-manifolds [12]; also, finite groups with balanced presentations (such finite groups have trivial Schur multiplier [13]). Finite metacyclic groups are efficient. This was shown by Beyl [6] and Wamsley [27]. Infinite metacyclic groups, however, need not be efficient, a result due to Baik and Pride [5] (see also [3]). In [13], Harlander proved that a finitely presented group embeds into an efficient group. In [16], Johnson 
showed that all finite $p$-groups are efficient under direct products and standard wreath products (for $p$ odd). Then, Wamsley [26] showed that all finite $p$-groups are efficient under general wreath products. For more references on the subject of efficiency see Baik and Pride [4], Beyl and Rosenberger [7], Campbell, Robertson and Williams [9] (and [10]), Harlander [14], Johnson and Robertson [17], Kenne [19], and Robertson, Thomas and Wotherspoon [23].

Not all finitely presented groups are efficient.

Neumann [22] asked whether a finite group $G$ with $\delta(G)=0$ must be efficient. Swan [25] gave examples (of finite metabelian groups) which showed this not to be the case. These were the first examples of inefficient groups. In [29], Wiegold produced a different construction to the same end, and then Neumann added a slight modification to reduce the number of generators. In [20], Kovacs generalized both the above constructions, and he showed how to construct more inefficient finite groups (including some perfect groups) whose Schur multiplicator is trivial. In [23], Robertson, Thomas and Wotherspoon examined a class of groups, orginally introduced by Coxeter. By using a symmetric presentation, they showed that groups in this class are inefficient. They also proved that every finite simple group can be embedded into a finite inefficient group.

Lustig [21] gave the first example of a torsion-free inefficient group. Other examples were found by Baik (see [3]), using generalized graph products. In [4], Baik and Pride gave sufficient conditions for a Coxeter group to be efficient. They also found a family of inefficient Coxeter groups $G_{n, k}(n \geqslant 4, k$ an odd integer). For a fixed $k$ :

$$
\operatorname{def}\left(G_{n, k}\right)-\delta\left(G_{n, k}\right) \stackrel{n}{\rightarrow} \infty .
$$

We remark that there is no algorithm to decide for any finitely presented group whether or not the group is efficient (see [1]).

\section{(c) The definition of standard wreath product}

Let $A$ and $B$ be finite groups with $A=\left\{a_{1}, a_{2}, \ldots, a_{l}\right\}$, say. Let $x$ be any element of $A$. Then

$$
a_{1} x, a_{2} x, \ldots, a_{l} x
$$

is a permutation of $a_{1}, a_{2}, \ldots, a_{l}$. So we can write $a_{1} x, a_{2} x, \ldots, a_{l} x$ as

$$
a_{\sigma_{x}(1)}, a_{\sigma_{x}(2)}, \ldots, a_{\sigma_{x}(l)},
$$

where $\sigma_{x}$ is a permutation of $1,2, \ldots, l$.

Let $K$ be the direct product of the number of $|A|$ copies of $B$, that is,

$$
K=B^{|A|}=B^{l}=\underbrace{B \times B \times \cdots \times B}_{(l \text { times })},
$$

and let $\left(b_{a_{1}}, b_{a_{2}}, \ldots, b_{a_{l}}\right)$ be a typical element of $K$. We have a homomorphism

$$
\theta: A \longrightarrow \operatorname{Aut}(K), \quad x \longmapsto \theta_{x},
$$


where

$$
\left(b_{a_{1}}, b_{a_{2}}, \ldots, b_{a_{l}}\right) \theta_{x}=\left(b_{a_{\sigma_{x}(1)}}, b_{a_{\sigma_{x}(2)}}, \ldots, b_{a_{\sigma_{x}(l)}}\right) .
$$

The split extension $K \rtimes_{\theta} A$ is called the standard wreath product of $B$ by $A$, denoted $B$ ? $A$. (We should note that some authors, for instance Karpilovsky in [18], use the notation $A$ < $B$ instead of $B \backslash A$. Here we use the notation as in [24]. Also, the definition of general wreath product, which will not be needed here, can be found in [24].)

(d) The main theorem

Let $A$ and $B$ be finite groups satisfying the following conditions.

(i) $A, B$ have efficient presentations $\mathcal{P}_{A}=\langle\boldsymbol{x} ; \boldsymbol{r}\rangle$ and $\mathcal{P}_{B}=\langle\boldsymbol{y} ; \boldsymbol{s}\rangle$, respectively, on $g$, $n(g, n \in \mathbb{N})$ generators, where $n=d(B)$.

(ii) $d(B)=d\left(H_{1}(B)\right)$.

(iii) Either

(a) the order of $A$ is even and also $t\left(H_{2}(A)\right), t\left(H_{2}(B)\right)$ and $t\left(H_{1}(B)\right)$ are all even;

or

(b) the order of $A$ is odd and there exists a prime $p$ dividing $t\left(H_{2}(A)\right), t\left(H_{2}(B)\right)$ and $t\left(H_{1}(B)\right)$, where $t(\cdot)$ is the first torsion number as defined in Definition 2.5.

Theorem 1.1 (Main Theorem). Let $G=B \imath A$, and suppose that (i), (ii) and (iii) hold. Then $G$ has an efficient presentation on $g+n$ generators.

Remark 1.2. The reason for us keeping track of the number of generators is that there is interest not just in finding efficient presentations, but in finding presentations that are efficient on the minimal number of generators (see [28]).

Remark 1.3. To prove our theorem, we will obtain from $\mathcal{P}_{A}, \mathcal{P}_{B}$ a 'canonical' presentation $\mathcal{P}_{3}$ for $G$. It will turn out that assuming (i) and (ii), condition (iii) is both necessary and sufficient for $\mathcal{P}_{3}$ to be efficient. We suspect, though cannot prove, that $\mathcal{P}_{3}$ is always minimal (that is, $\operatorname{def}\left(\mathcal{P}_{3}\right)=\operatorname{def}(G)$ ). See Example 4.6 for some simple examples when $\mathcal{P}_{3}$ is not efficient.

Remark 1.4. After this paper was submitted it was brought to our attention that a special case of our theorem was obtained independently in [2].

\section{Preliminary material}

Proposition 2.1 (Schur 1904). Let $B$ be a finite group. Then

(i) $\mathrm{H}_{2}(B)$ is a finite group, whose elements have order dividing the order of $B$;

(ii) $H_{2}(B)=1$ if $B$ is cyclic. 


\section{Definition 2.2.}

(1) Given an abelian group $A$, we denote by $A \# A$ the factor group of $A \otimes A$ by the subgroup generated by the elements of the form $a \otimes b+b \otimes a,(a, b \in A)$.

(2) In any group $K$, an element of order 2 is called an involution.

Theorem 2.3 (Blackburn 1972). Let $m$ denote the number of involutions in the group $A$. Then

$$
\begin{array}{r}
H_{2}(B \backslash A)=H_{2}(B) \oplus H_{2}(A) \oplus\left(H_{1}(B) \otimes H_{1}(B)\right)^{(1 / 2)(|A|-m-1)} \\
\oplus\left(H_{1}(B) \# H_{1}(B)\right)^{m} .
\end{array}
$$

Let $\mathbb{Z}_{n}$ denote the cyclic group of order $n$.

Lemma 2.4. Let $B$ be a finite group, let

$$
H_{1}(B) \cong \bigoplus_{i=1}^{t} \mathbb{Z}_{n_{i}}
$$

and let $s$ be the number of even $n_{i}, 1 \leqslant i \leqslant t$. Then

$$
H_{1}(B) \# H_{1}(B) \cong \bigoplus_{1 \leqslant i<j \leqslant t} \mathbb{Z}_{\left(n_{i}, n_{j}\right)} \oplus \mathbb{Z}_{2}^{s},
$$

where $\mathbb{Z}_{2}^{s}$ is a direct sum of $s$ copies of $\mathbb{Z}_{2}$.

Proofs of Proposition 2.1, Theorem 2.3 and Lemma 2.4 can be found in [18].

Definition 2.5. Let $A$ be a non-trivial finite abelian group. Then (see [24]) $A$ can be uniquely written as

$$
A=\mathbb{Z}_{n_{1}} \oplus \mathbb{Z}_{n_{2}} \oplus \cdots \oplus \mathbb{Z}_{n_{r}}, \quad n_{1}\left|n_{2}\right| \cdots \mid n_{r} .
$$

We define $t(A)$ to be $n_{1}$. If $A=0$, we define $t(A)$ to be 0 .

The proof of the following lemma can be found, for instance, in [11].

Lemma 2.6. Let $A$ and $B$ be finite abelian groups. If $(t(A), t(B)) \neq 1$, then

$$
d(A \oplus B)=d(A)+d(B) .
$$

It is clear that the above lemma can be generalized for more than two abelian groups.

\section{Proof of the main theorem}

Throughout this section, $A$ and $B$ will be finite groups satisfying conditions (i), (ii) and (iii), and $m$ will denote the number of involutions in $A$.

In this part of the proof, we will calculate $\delta(G)$ as given in (1.1). Now, since $G$ is a finite group, $r k_{\mathbb{Z}}\left(H_{1}(G)\right)=0$, so we will just calculate $\delta(G)=d\left(H_{2}(G)\right)$. Recall that we had the formula (2.1) to calculate $H_{2}(G)$. 
Let us write

$$
\begin{array}{lll}
H_{1}(B)=\mathbb{Z}_{v_{1}} \oplus \mathbb{Z}_{v_{2}} \oplus \cdots \oplus \mathbb{Z}_{v_{n}}, & v_{i} \mid v_{i+1}, & 1 \leqslant i \leqslant n-1, \\
H_{2}(B)=\mathbb{Z}_{k_{1}} \oplus \mathbb{Z}_{k_{2}} \oplus \cdots \oplus \mathbb{Z}_{k_{q}}, & k_{i} \mid k_{i+1}, & 1 \leqslant i \leqslant q-1, \\
H_{2}(A)=\mathbb{Z}_{l_{1}} \oplus \mathbb{Z}_{l_{2}} \oplus \cdots \oplus \mathbb{Z}_{l_{r}}, & l_{i} \mid l_{i+1}, & 1 \leqslant i \leqslant r-1
\end{array}
$$

By (ii), $d\left(H_{1}(B)\right)=d(B)=n$.

We have

$$
H_{1}(B) \otimes H_{1}(B)=\bigoplus_{i=1}^{n} \mathbb{Z}_{v_{i}}^{2(n-i)+1}
$$

Suppose $|A|$ is even. Then, by (iii) (a), $v_{1}$ is even and so by (2.2)

$$
H_{1}(B) \# H_{1}(B)=\bigoplus_{i=1}^{n} \mathbb{Z}_{v_{i}}^{(n-i)} \oplus \mathbb{Z}_{2}^{s}
$$

Using (2.1) and Lemma 2.6, we then get

$$
\begin{aligned}
d\left(H_{2}(G)\right)= & d\left(H_{2}(A)\right)+d\left(H_{2}(B)\right) \\
& +\frac{1}{2}(|A|-m-1) \sum_{i=1}^{n}(2(n-i)+1)+m\left(\sum_{i=1}^{n}(n-i)+n\right) \\
= & d\left(H_{2}(A)\right)+d\left(H_{2}(B)\right)+\frac{1}{2} d(B)^{2}\left(|A|-1+\frac{m}{d(B)}\right) .
\end{aligned}
$$

Notice that if (iii) (a) fails, then either $v_{1}$ is odd (in which case $s<n$ ), or $v_{1}$ is even (in which case $s=n$ and one of $k_{1}, l_{1}$, say $k_{1}$, must be odd, so the cyclic group $\mathbb{Z}_{k_{1}} \oplus \mathbb{Z}_{2}$ occurs in the direct sum equation (2.1)). Thus, the equality in (3.1) becomes a strict inequality $<$.

On the other hand, if $|A|$ is odd, then $m=0$ and, by assuming that (iii) (b) holds, a similar calculation shows that the formula (3.1) is still valid. Moreover if (iii) (b) fails then either $q, r>0$ and the 2-generator group $\mathbb{Z}_{k_{1}} \oplus \mathbb{Z}_{l_{1}} \oplus \mathbb{Z}_{v_{1}}$ occurs in the direct sum equation (2.1), or one of $q, r$, say $q$, is 0 , and the cyclic group $\mathbb{Z}_{v_{1}} \oplus \mathbb{Z}_{l_{1}}$ occurs in (2.1), so again (3.1) becomes a strict inequality $<$.

In this part of the proof, we need to obtain an efficient presentation for $G=B \imath A$. The following process can be followed.

(i) For each $a \in A$, take a copy $\left\langle y^{(a)} ; \boldsymbol{s}^{(a)}\right\rangle$ of $\mathcal{P}_{B}$.

(ii) Choose an ordering $a_{1}<a_{2}<\cdots<a_{n}$ of the elements of $A$ where $a_{1}=1$.

(iii) Let $\left\{a_{x}: x \in \boldsymbol{x}\right\}$ be a generating set for $A$ corresponding to the presentation $\mathcal{P}_{A}=\langle\boldsymbol{x} ; \boldsymbol{r}\rangle$.

(iv) Let $\left\{b_{y}: y \in y\right\}$ be a generating set for $B$ corresponding to the presentation $\mathcal{P}_{B}=\langle\boldsymbol{y} ; \boldsymbol{s}\rangle$. 
A presentation of $G=B\} A$ is then given by

$$
\begin{aligned}
\mathcal{P}_{1}=\left\langle\boldsymbol{y}^{(a)}(a \in A), \boldsymbol{x} ; \boldsymbol{s}^{(a)}(a \in A),\right. & \\
\boldsymbol{r}, \boldsymbol{y}^{(a)} z^{\left(a^{\prime}\right)}=z^{\left(a^{\prime}\right)} y^{(a)} & \left(a, \boldsymbol{a}^{\prime} \in A, a<a^{\prime}, y, z \in \boldsymbol{y}\right), \\
& \left.x^{-1} y^{(a)} x=y^{\left(a a_{x}\right)}(a \in A, y \in \boldsymbol{y}, x \in \boldsymbol{x})\right\rangle .
\end{aligned}
$$

In $[\mathbf{1 5}$, ch. 15], it is shown how to simplify this presentation, as follows. The set $A \backslash\{1\}$ can be divided into singletons $\{a\}\left(a \in A, a\right.$ an involution) and pairs $\left\{a, a^{-1}\right\}$ ( $a$ not an involution). Let $A^{+}$be a choice of one element from each pair $\left\{a, a^{-1}\right\}$. (Note that $\left|A^{+}\right|=\frac{1}{2}(|A|-1-m)$.) Let Inv be the set of the involutions in the group $A$. Then

$$
\mathcal{P}_{2}=\left\langle\boldsymbol{y}, \boldsymbol{x} ; \boldsymbol{s}, \boldsymbol{r},\left[y, W_{a}^{-1} z W_{a}\right]\left(a \in A^{+} \cup \operatorname{Inv}, y, z \in \boldsymbol{y}\right)\right\rangle
$$

is a presentation for $B \backslash A$. Here, $W_{a}$ is a word on $\boldsymbol{x}$ representing $a$.

Now, we can still apply some reductions on the relators $\left[y, W_{a}^{-1} z W_{a}\right]\left(a \in A^{+} \cup \operatorname{Inv}\right.$, $y, z \in \boldsymbol{y})$. Note that the number of these relators is

$$
\frac{1}{2}(|A|-1+m)|\boldsymbol{y}|^{2} .
$$

Let us choose an ordering $y_{1}<y_{2}<\cdots_{i}<y_{n}$ of the elements of the generating set $\boldsymbol{y}$. Then we can delete the relators of the form $\left[z, W_{a}^{-1} y W_{a}\right](a \in \operatorname{Inv}, y, z \in y, y<z)$, since these are consequences of the relators of the form $\left[y, W_{a}^{-1} z W_{a}\right](a \in \operatorname{Inv}, y, z \in \boldsymbol{y}$, $y<z)$, as is shown as follows. Let $a \in \operatorname{Inv}$ and $y, z \in \boldsymbol{y}$, where $y<z$. Let us take a relator $\left[y, W_{a}^{-1} z W_{a}\right]$, and let us conjugate it by $W_{a}$. Then we get $\left[W_{a} y W_{a}^{-1}, z\right]$. The inverse of it is $\left[z, W_{a} y W_{a}^{-1}\right]$. But, since $a \in \operatorname{Inv}$, we have $W_{a}=W_{a}^{-1}$ in $A$. So, we get $\left[z, W_{a}^{-1} y W_{a}\right]$, as required.

Then we have the presentation

$$
\begin{aligned}
\mathcal{P}_{3}=\left\langle\boldsymbol{y}, \boldsymbol{x} ; \boldsymbol{s}, \boldsymbol{r},\left[y, W_{a}^{-1} z W_{a}\right]\left(a \in A^{+}, \boldsymbol{y}, z \in \boldsymbol{y}\right),\right. & \\
& {\left.\left[y, W_{a}^{-1} z W_{a}\right](a \in \mathrm{Inv}, y, z \in \boldsymbol{y}, y \leqslant z)\right\rangle . }
\end{aligned}
$$

Now the number of relators $\left[y, W_{a}^{-1} z W_{a}\right]\left(a \in A^{+}, y, z \in y\right)$ is $\frac{1}{2}(|A|-1-m)|\boldsymbol{y}|^{2}$ and the number of relators $\left[y, W_{a}^{-1} z W_{a}\right](a \in \operatorname{Inv}, y, z \in \boldsymbol{y}, y \leqslant z)$ is $m|\boldsymbol{y}|^{2}-\frac{1}{2}|\boldsymbol{y}|(|\boldsymbol{y}|-1) m$. So we have in total

$$
\frac{1}{2}|\boldsymbol{y}|^{2}(|A|-1+(m /|\boldsymbol{y}|))
$$

commutator relators in $\mathcal{P}_{3}$.

If (iii) holds, then, by using (3.1) and the fact that

$$
d\left(H_{2}(A)\right)=-|\boldsymbol{x}|+|\boldsymbol{r}|, \quad d\left(H_{2}(B)\right)=-|\boldsymbol{y}|+|\boldsymbol{s}|
$$

(since $\mathcal{P}_{A}, \mathcal{P}_{B}$ are efficient presentations), we easily find that

$$
\operatorname{def}\left(\mathcal{P}_{3}\right)=d\left(H_{2}(G)\right)
$$

and so $\mathcal{P}_{3}$ is an efficient presentation for $G$. 
Suppose that $g=d(A),\left(t\left(H_{1}(A)\right), t\left(H_{1}(B)\right)\right) \neq 1$ and $d\left(H_{1}(A)\right)=d(A)$. Since $\mathcal{P}_{3}$ has $g+n$ generators, then we certainly have $d(G) \leqslant g+n$. Also, by the fact $d(G) \geqslant d\left(G^{a b}\right)$, we need to get $d\left(G^{a b}\right)=g+n$. Now let us choose an ordering $x_{1}<x_{2}<\cdots<x_{g}$ of the elements of the generating set $\boldsymbol{x}$. Then it is easy to see that

$$
\begin{aligned}
G^{a b}= & \langle\boldsymbol{y}, \boldsymbol{x} ; \boldsymbol{s}, \boldsymbol{r},[y, z](y, z \in \boldsymbol{y}, y<z), \\
& {\left.\left[x, x^{\prime}\right]\left(x, x^{\prime} \in \boldsymbol{x}, x<x^{\prime}\right),[y, x](y \in \boldsymbol{y}, x \in \boldsymbol{x})\right\rangle } \\
\cong & A^{a b} \oplus B^{a b}=H_{\mathbf{1}}(A) \oplus H_{1}(B) .
\end{aligned}
$$

Thus, by (ii) and Lemma 2.6, we get $d\left(G^{a b}\right)=g+n$, as required.

Notice that if condition (iii) fails, then, from our previous discussion,

$$
\operatorname{def}\left(\mathcal{P}_{3}\right)>d\left(H_{2}(G)\right)
$$

and so $\mathcal{P}_{3}$ is not efficient.

\section{Examples and applications}

In this section we give some examples and applications of Theorem 1.1.

Example 4.1. Let $A$ be a finite group and $B$ be the metacyclic group of order 20 defined by the presentations $\mathcal{P}_{A}$ and $\mathcal{P}_{B}=\left\langle a, b ; a^{10}, b^{2}, b a b^{-1}=a^{-1}\right\rangle$, respectively. Then we have the presentation $\mathcal{P}_{3}$ for $B$ \} $A$.

Suppose $\mathcal{P}_{A}$ is efficient. By [18], $H_{2}(B)=\mathbb{Z}_{2}$, so $\mathcal{P}_{B}$ is also efficient. Then condition (i) holds. Also, a simple calculation shows that $H_{1}(B) \cong \mathbb{Z}_{2} \times \mathbb{Z}_{2}$. So, $d(B)=2=d\left(H_{1}(B)\right)$ and then condition (ii) holds.

Thus, since $t\left(H_{2}(B)\right)=2=t\left(H_{1}(B)\right)$, if $|A|$ is even and $2 \mid t\left(H_{2}(A)\right)$, then the presentation $\mathcal{P}_{3}$ for $B \backslash A$ is efficient. Additionally, if $\mathcal{P}_{A}$ is an efficient presentation on $g=d(A)=d\left(H_{1}(A)\right)$ generators and $2 \mid t\left(H_{1}(A)\right)$, then $\mathcal{P}_{3}$ is an efficient presentation on $d(B \imath A)=2+g$ generators.

Example 4.2. Now, let $A, B$ be finite groups defined by the presentations $\mathcal{P}_{A}$ and $\mathcal{P}_{B}=\left\langle a, b ; a^{3}, b^{3},(a b)^{3},\left(a^{-1} b\right)^{3}\right\rangle$, respectively. We then have the presentation $\mathcal{P}_{3}$ for $\left.B\right\} A$.

Suppose $\mathcal{P}_{A}$ is efficient. By [18], $B$ has order 27 and $H_{2}(B)=\mathbb{Z}_{3} \times \mathbb{Z}_{3}$. Thus $\mathcal{P}_{B}$ is an efficient presentation of $B$. So condition (i) holds. One can find $H_{1}(B) \cong \mathbb{Z}_{3} \times \mathbb{Z}_{3}$. Then $d(B)=2=d\left(H_{1}(B)\right)$, so condition (ii) holds.

Also, since $t\left(H_{2}(B)\right)=3=t\left(H_{1}(B)\right)$, if $|A|$ is odd and $3 \mid t\left(H_{2}(A)\right)$, then the presentation $\mathcal{P}_{3}$ for $B$ ? $A$ is efficient. Moreover, if $\mathcal{P}_{A}$ is efficient on $g=d(A)=d\left(H_{1}(A)\right)$ generators and $3 \mid t\left(H_{1}(A)\right)$, then $\mathcal{P}_{3}$ is an efficient presentation on $d(B, A)=2+g$ generators.

The proof of the following proposition can be found, for instance, in [11].

Proposition 4.3. Let $B$ be an arbitrary finite p-group. Then

$$
d(B)=d\left(H_{1}(B)\right)
$$


Corollary 4.4. Let $A, B$ be finite $p$-groups. Suppose $B$ has an efficient presentation on $d(B)$ generators and $A$ has an efficient presentation. Then $B \backslash A$ has an efficient presentation. Moreover, if $A$ has an efficient presentation on $d(A)$ generators, then $B$ r $A$ has an efficient presentation on $d(B<A)$ generators.

Proof. It is given that $A$ has an efficient presentation and $B$ has an efficient presentation on $d(B)$ generators. Since they are finite $p$-groups then, by Proposition 4.3 , $d(B)=d\left(H_{1}(B)\right), d(A)=d\left(H_{1}(A)\right)$ and their homology groups are $p$-groups as well. So $p$ divides $t\left(H_{2}(B)\right), t\left(H_{2}(A)\right), t\left(H_{1}(B)\right)$ and $t\left(H_{1}(A)\right)$. Now suppose that the efficient presentation of $A$ is on $d(A)$ generators. Then, by Theorem 1.1,B $>A$ has an efficient presentation on $d(B \backslash A)=d(B)+d(A)$ generators, as required.

The following result can be proved as Corollary 4.4 .

Corollary 4.5. Let $A$ be a finite group and $B$ be a finite $p$-group for any prime $p$. Suppose that $B$ has an efficient presentation on $d(B)$ generators and $A$ has an efficient presentation. If $p$ divides $t\left(H_{2}(A)\right)$ then $B ? A$ has an efficient presentation. Moreover, if $A$ has an efficient presentation on $d(A)$ generators such that $d(A)=d\left(H_{1}(A)\right)$ and $p$ divides $t\left(H_{1}(A)\right)$, then $B ? A$ has an efficient presentation on $d(B \backslash A)$ generators.

In the following example, we give some cases when the presentation $\mathcal{P}_{3}$ for $B$ ? $A$ is not efficient.

Example 4.6. Let $A=\mathbb{Z}_{m} \times \mathbb{Z}_{m}$ and $B=\mathbb{Z}_{k}$, which are defined by the presentations $\mathcal{P}_{A}=\left\langle x, \bar{x} ; x^{m}, \bar{x}^{m},[x, \bar{x}]\right\rangle$ and $\mathcal{P}_{B}=\left\langle y ; y^{k}\right\rangle$, respectively. We then have the presentation $\mathcal{P}_{3}$ for $\left.B\right\} A$.

By Kunneth formula, we get $H_{2}(A)=\mathbb{Z}_{m}$. Thus, $\mathcal{P}_{A}$ is an efficient presentation for $A$. On the other hand, by Proposition $2.1, H_{2}(B)=1$ so $\mathcal{P}_{B}$ is an efficient presentation for $B$. Thus, condition (i) holds. Notice that $H_{1}(B)=\mathbb{Z}_{k}$, so $1=d(B)=d\left(H_{1}(B)\right)$ and then condition (ii) holds.

Now it is easy to see that if $m$ is even and $k$ is odd then condition (iii) (a) fails. Similarly, if $m$ is odd and $m, k$ are coprime, then (iii) (b) fails. Therefore, $\mathcal{P}_{3}$ is not efficient.

Question. For $A, B$ as above, is $\mathcal{P}_{3}$ minimal?

Acknowledgements. I express my deepest thanks to Professor S. J. Pride for suggesting this work to me, and for his guidance.

\section{References}

1. A. G. B Aнmad, The unsolvability of efficiency for groups, Southeast Asian Bull. Math. 22 (1998), 331-336.

2. H. Ayik, C. M. Campbell, J. J. O'Connor and N. RušKuC, On the efficiency of wreath products of groups, Preprint, University of St Andrews (1998).

3. Y. G. BAIK, Generators of the second homotopy module of group presentations with applications, PhD thesis, University of Glasgow (1992).

4. Y. G. BAIK AND S. J. Pride, On the efficiency of Coxeter groups, Bull. Lond. Math. Soc. 29 (1997), 32-36.

5. Y. G. BAIK AND S. J. PRIDE, Generators of second homotopy modules of presentations arising from group constructions, Preprint, University of Glasgow (1993). 
6. F. R. BEYL, The Schur-multiplicator of metacyclic groups, Proc. Am. Math. Soc. 40 (1973), 413-418.

7. F. R. BEYL AND G. Rosenberger, Efficient presentations of $G L(2, \mathbb{Z})$ and $P G L(2, \mathbb{Z})$ (ed. E. F. Robertson and C. M. Campbell), Groups-St Andrews 1985, London Mathematical Society Lecture Notes, vol. 121, pp. 135-137 (Cambridge University Press, 1986).

8. F. R. BEYL AND J. TAPPE, Group extensions, representations and the Schur multiplicator, Lecture Notes in Mathematics, vol. 958 (Springer, 1982).

9. C. M. Campbell, E. F. Robertson And P. D. Williams, Efficient presentations for finite simple groups and related groups (ed. A. C. Kim and B. H. Neumann), GroupsKorea 1988, Lecture Notes in Mathematics, vol. 1398, pp. 65-72 (Springer, 1989).

10. C. M. Campbell, E. F Robertson and P. D. Williams, On the efficieny of some direct powers of groups (ed. L. G. Kovacs), Groups-Canberra 1989, Lecture Notes in Mathematics, vol. 1456, pp. 106-113 (Springer, 1990).

11. A. S. ÇEvIK, Minimality of group and monoid presentations, PhD thesis, University of Glasgow (1997).

12. D. B. A. EPSTEIn, Finite presentations of groups and 3-manifolds, Q. J. Math. Oxford 12 (1961), 205-212.

13. J. Harlander, Embeddings into efficient groups, Proc. Edinb. Math. Soc. 40 (1997), 313-324.

14. J. HaRlander, Closing the relation gap by direct-product stabilization, J. Alg. 182 (1996), 511-521.

15. D. L. JOHNSON, Presentation of groups, London Mathematical Society Lecture Notes Series, vol. 22 (Cambridge University Press, 1976).

16. D. L. Johnson, Minimal relations for certain wreath products of groups, Can. J. Math. XXII (1970), 1005-1009.

17. D. L. JohnSON AND E. F. ROBERTSON, Finite groups of deficiency zero, in Homological group theory (ed. C. T. C. Wall), London Mathematical Society Lecture Notes Series, vol. 36, pp. 275-289 (Cambridge University Press, 1979).

18. G. KARPILOVSKY, The Schur multiplier, London Mathematical Society Monograms New Series 2 (Oxford Science Publications, 1987).

19. P. E. Kenne, Some new efficient soluble groups, Comm. Alg. 18 (1990), 2747-2753.

20. L. G. KovaCS, Finite groups with trivial multiplicator and large deficiency (ed. A. C. Kim and D. L. Johnson), Groups-Korea 1994, pp. 277-284 (Walter de Gruyter, 1995).

21. M. LUSTIG, Fox ideals, $\mathcal{N}$-torsion and applications to groups and 3-manifolds, in Twodimensional homotopy and combinatorial group theory (ed. C. Hog-Angeloni, W. Metzler and A. J. Sieradski), pp. 219-250 (Cambridge University Press, 1993).

22. B. H. Neumann, Some groups with trivial multiplicators, Publ. Math. Debrecen 4 (1955), 190-194.

23. E. F. Robertson, R. M. Thomas And C. I. Wotherspoon, A class of inefficient groups with symmetric presentations (ed. A. C. Kim and D. L. Johnson), Groups-Korea 1994 (Walter de Gruyter, 1995).

24. J. J. Rotman, Theory of groups, 3rd edn (W. C. Brown, Iowa, 1988).

25. R. G. SWAN, Minimal resolutions for finite groups, Topology 4 (1965), 193-208.

26. J. W. WAMSLEY, The deficiency of wreath products of groups, J. Alg. 27 (1973), 48-56.

27. J. W. Whasley, The deficiency of metacyclic groups, Proc. Am. Math. Soc. 24 (1970), 724-726.

28. J. W. WAMSLEY, Minimal presentations for finite groups, Bull. Lond. Math. Soc. 5 (1973), 129-144.

29. J. WIEGOLD, The Schur multiplier: an elementary approach (ed. C. M. Campbell and E. F. Robertson), Groups-St Andrews 1981, London Mathematical Society Lecture Note Series, vol. 71, pp. 137-154 (Cambridge University Press, 1981). 